\title{
Stability of nanobeams and nanoplates with defects
}

\author{
Hina ARIF AND JAAN LELleP
}

\begin{abstract}
The sensitivity of critical buckling load and critical stress concerning different geometrical and physical parameters of Euler-Bernoulli nanobeams with defects is studied. Eringen's nonlocal theory of elasticity is used for the determination of critical buckling load for stepped nanobeams subjected to axial loads for different support conditions. An analytical approach to study the impact of discontinuities and boundary conditions on the critical buckling load and critical stress of nanobeams has been developed. Critical buckling loads of stepped nanobeams are defined under the condition that the nanoelements are weakened with stable crack-like defects. Simply supported, clamped and cantilever nanobeams with steps and cracks are investigated in this article. The presented results are compared with the other available results and are found to be in a close agreement.
\end{abstract}

\section{Introduction}

Nanomaterials are of great importance in the field of physics, chemistry and engineering. Because of the special mechanical, electronic and electrical properties, nanomaterials are the fundamental components of various nanocomposites and nanoelectromechanical systems. Some well-known examples of nanomaterials are nanoparticles, nanotubes and nanowires. Beamlike nanostructures are widely used in civil, mechanical and aerospace engineering [3]. Nanobeam plays an important role in the field of nanotechnology.

Because of the various practical applications, static and dynamic analysis of nanobeams have been carried out by the researchers. The experimental and atomistic simulation results indicate that the small length scale may not be neglected at nanoscale. Due to this reason, the classical local continuum

Received February 14, 2021.

2020 Mathematics Subject Classification. 74B20.

Key words and phrases. Buckling, stepped nanobeam, crack, critical buckling load. https://doi.org/10.12697/ACUTM.2021.25.15

Corresponding author: Hina Arif 
theories fail to analyse the nanostructures. So the use of nonlocal theories like strain gradient theory, couple stress theory, modified couple stress theory also called Eringen's elasticity theory is necessary to analyse nanoscale structures.

In the present paper, Eringen's nonlocal theory of elasticity is used to analyse the stability of Euler-Bernoulli nanobeams. Eringen [14, 15] developed a promising theory to investigate the nanomaterials without ignoring the internal length scale and accounts for the forces acting between atoms. Static and dynamic problems of stability were successfully carried out by using nonlocal theory of elasticity. Some of the dynamic problems involving the determination of frequencies of natural vibrations of nanobeams/rods are carried out by Bagdatli [9], Lellep and Lenbaum [20], Roostai and Haghpanahi [27], Lu et al. [24] and also by Li et al. [23]. The buckling analysis of nanobeams/rods was carried out with the help of nonlocal theory of elasticity by Adali [1], Emam [13], Reddy [29, 30], Challamel et al. [10, 11] and others.

Wang et al. [33, 34, 35] applied Timoshenko beam theory for the buckling analysis of micro and nanorods. Similarly Murma and Pradhan [25] investigated carbon nanotubes embedded in an elastic medium by using Timoshenko beam theory. A review article on the behaviour of thin-walled carbon nanotubes is presented by Arash and Wang [4]. The Euler-Bernoulli, Timoshenko, Reddy and Levinson beam theories were reformulated by Reddy [30]. Reddy [29] also used Eringen's nonlocal constitutive relations to analyse static and dynamic stability of beams and plates with different boundary conditions. Lu et al. [24], Thai [32], Reddy [29], Li et al. [23] and Wang et al. [35] used the analytical approach to analyse the dynamic behaviour of nanobeams. Ansari and Sahmoni [2] presented the comparison of different beam theories applied to the anlysis of nanobeams. Wang at al. [35], Zhang et al. [36], and Kumar et al. [18] examine the stability of nanobeams under conservative loading and analyse the impact of nonlocal parameters and the influence of different boundary conditions on the critical buckling loads and postcritical states of nanobeams.

Since conducting experiments at nanolevel is difficult to handle, the mathematical modelling plays an important role in the field of nanotechnology. Efforts have been made by researchers to develop efficient analytical and numerical techniques for obtaining the desired results. In the present paper, an analytical approach is developed to study the influence of physical and geometrical parameters on the stability of nanobeams/plates. Stepped nanobeams of rectangular cross-section weakened with crack-like defects are considered here. An attempt has been made to study the influence of cracks on the buckling analysis of nanobeams/plates. Nanobeams with different 
support conditions are investigated in the present paper. The obtained results are found to be in good agreement with the existing results of other researchers.

\section{Model description}

Consider a nanobeam/plate of length $l$ under the axial compression $P$. The coordinates are introduced in such a manner that the origin is considered to be at the centre of the left-hand edge of the nanobeam. A nanobeam/plate having a step at $x=a_{k}, k=1, \ldots, n$, is investigated in the present paper. It is assumed that the nanobeam/plate has a rectangular cross-section with the width $b=$ constant and the height $h$ as

$$
h=h_{k}=\text { const, for } x \in\left(a_{k}, a_{k+1}\right), k=0, \ldots, n .
$$

The quantities $a_{k}$ are assumed to be constants, with $a_{0}=0, a_{n+1}=l$. It is assumed that the nanobeam of length $l$ has a defect at the step-location $x=a_{k}, k=1, \ldots, n$. The defect is considered to be a stable surface crack of length $c_{k}$, which is assumed to be uniformly penetrated throughout the width of the nanobeam as shown in Figure 1.

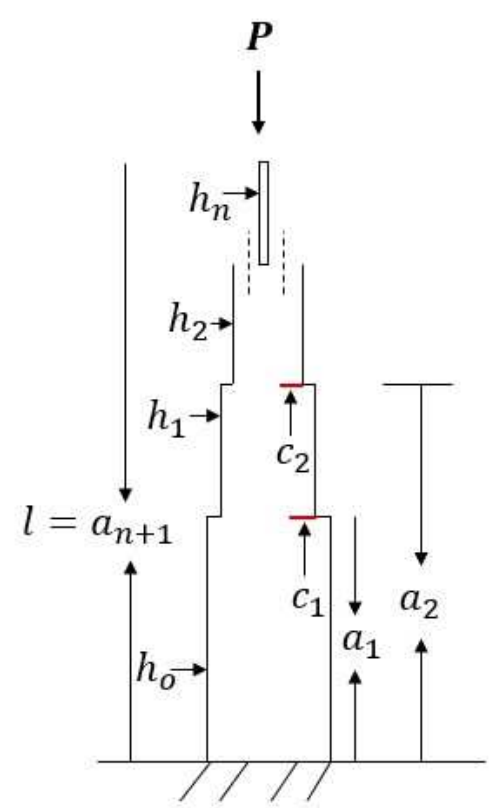

FigURE 1. n-stepped nanobeam with cracks. 
The aim of the present paper is to determine the critical buckling loads of stepped nanobeams weakened by cracks and to investigate the influence of discontinuities like cracks, steps and various boundary conditions on the critical buckling loads of nanobeams. Similarly the sensitivity of critical buckling loads to some other physical and geometrical parameters is also studied. Simply supported, clamped and cantilever nanobeams/plates (see Figure 2) are investigated here.

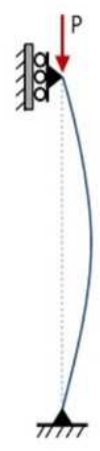

(a) Simply supported nanobeam

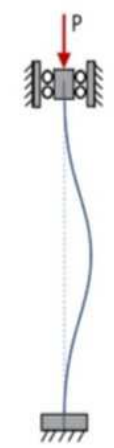

(b) Clamped nanobeam

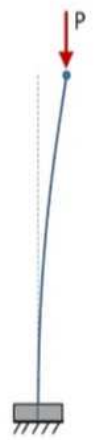

(c) Cantilever nanobeam

FiguRE 2. Nanobeams with different support conditions.

\section{Nonlocal constitutive equations for Euler-Bernoulli nanobeams}

According to Eringen's nonlocal theory of elasticity [14, 15], the stress state at a reference point $x$ in a continuum medium depends not only on the strain state at $x$ but also on the strain states at all other points $x^{\prime}$ of the medium. The constitutive equation in the nonlocal elasticity can be presented as (see $[2,7,14,15,20,26,29])$

$$
\sigma_{i j}(x)=\int_{V} K\left(\left|x-x^{\prime}\right|, \tau\right) t_{i j} d V\left(x^{\prime}\right), \quad \forall x \in V .
$$

Here $K$ is the nonlocal kernel function which shows the effect of the strain at the point $x^{\prime}$ on the stress at the point $x$. In (2) the quantities $\sigma_{i j}$ and $t_{i j}$ represent the nonlocal stress tensor and the local stress tensor, respectively. The integration covers the entire volume $V$ of the medium, $\tau$ is the scale effect which is based on the internal and external length characteristics and is defined as

$$
\tau=\frac{e_{o} a}{\bar{l}}
$$


In (3) $e_{o}$ is the material constant, $a$ is the lattice parameter and $\bar{l}$ is the size of the sample. A simplified version of the nonlocal constitutive relations for Euler-Bernoulli nanobeams can be described as (see [14, 15, 20, 26, 29])

$$
\sigma_{x x}-\left(e_{o} a\right)^{2} \frac{\partial^{2}}{\partial x^{2}} \sigma_{x x}=E \varepsilon_{x x},
$$

where $\varepsilon_{x x}$ is the normal strain and $E$ represents the Young's modulus of elasticity. Equation (4) can be written in terms of the bending moment $M$ as

$$
M-\eta \frac{\partial^{2} M}{\partial x^{2}}=M_{c}
$$

where $\eta=\left(e_{o} a\right)^{2}$ is the nonlocal length scale parameter and the value of $e_{o} a \leq 2 \mathrm{~nm}$. Here $M_{c}$ is the value of the bending moment in the classical theory of elasticity and can be defined, as in [33], by

$$
M_{c}=-E I_{k} \frac{\partial^{2} w}{\partial x^{2}},
$$

where $I_{k}$ represents the second moment of the cross-section. For a beam of rectangular cross-section having width $b$ and the height $h_{k}$ as defined by (1), one has

$$
I_{k}=\frac{b h_{k}^{3}}{12}, k=0, \ldots, n .
$$

In (6) $w$ stands for the lateral displacement. The bending moment $M$ for nonlocal theory of elasticity can be calculated by substituting (5) in (6). This yields finally

$$
M=-\left(E I_{k}-\eta P\right) w^{\prime \prime}
$$

for $x \in\left(a_{k}, a_{k+1}\right), k=0, \ldots, n$. It should be mentioned that $P$ is the compressive load and is treated here as a positive constant. Then the equilibrium conditions for the Euler-Bernoulli nanobeams have the form ([16], [22]),

$$
\begin{gathered}
Q=M^{\prime}, \\
Q^{\prime}=P w^{\prime \prime},
\end{gathered}
$$

where $Q$ represents the shear force. Eliminating $Q$ from (9) and (10) results in

$$
M^{\prime \prime}-P w^{\prime \prime}=0 .
$$

Substituting $M$ from (8) to (11) yields a fourth order ordinary differential equation with respect to the lateral displacement $w$ for $x \in\left(a_{k}, a_{k+1}\right)$ as

$$
w^{I V}+\lambda_{k}^{2} w^{\prime \prime}=0
$$

where

$$
\lambda_{k}=\left(\frac{P}{E I_{k}-\eta P}\right)^{\frac{1}{2}}, k=0, \ldots, n .
$$


The general solution of (12) can be presented as

$$
w=A_{k} \cos \lambda_{k} x+B_{k} \sin \lambda_{k} x+C_{k} x+D_{k},
$$

for $x \in\left(a_{k}, a_{k+1}\right), k=0, \ldots, n$. Here $A_{k}, B_{k}, C_{k}$ and $D_{k}$ are integration constants.

While constructing the solutions of the stability problems of nanobeams, we have to fit together the solutions of (14) for segments $\left(a_{k}, a_{k+1}\right)$ so that the corresponding continuity and jump conditions along with the boundary requirements are satisfied. For the nanobeam clamped at both ends, the transverse displacement $w$ and the slope of the transverse displacement $w^{\prime}$ must vanish at both ends. In such a case

$$
\begin{gathered}
w(0)=w^{\prime}(0)=0, \\
w(l)=w^{\prime}(l)=0 .
\end{gathered}
$$

In the case of a nanobeam simply supported at both ends the transverse displacement $w$ and the bending moment $M$ must vanish at both ends. Taking (8) into account one can state that in this case

$$
\begin{gathered}
w(0)=w^{\prime \prime}(0)=0, \\
w(l)=w^{\prime \prime}(l)=0 .
\end{gathered}
$$

However, in the case of a cantilever nanobeam one has

$$
\begin{gathered}
w(0)=w^{\prime}(0)=0, \\
M(l)=0, \quad Q(l)=P w^{\prime}(l) .
\end{gathered}
$$

\section{Continuity conditions and the local compliance}

The physical considerations show that the displacement $w=w(x)$, the bending moment $M$ and the shear force $Q$ are continuous everywhere, in particular at $x=a_{k}, k=1, \ldots, n$. Therefore,

$$
w\left(a_{k}-0\right)=w\left(a_{k}+0\right),
$$

similarly for the bending moment $M$ and the shear force $Q$, making use of (8) and (9) one can assert that

$$
\left(E I_{k-1}-\eta P\right) w^{\prime \prime}\left(a_{k}-0\right)=\left(E I_{k}-\eta P\right) w^{\prime \prime}\left(a_{k}+0\right),
$$

and

$$
\left(E I_{k-1}-\eta P\right) w^{\prime \prime \prime}\left(a_{k}-0\right)=\left(E I_{k}-\eta P\right) w^{\prime \prime \prime}\left(a_{k}+0\right),
$$

for every $k=1, \ldots, n$. However, according to Dimarogonas et al. [12] and Wang et al. [34], the slope $w^{\prime}$ has finite jumps passing the cross sections with stable cracks. The cracks are treated with the help of the rotating spring model [12]. Denoting

$$
\theta_{k}=w^{\prime}\left(a_{k}+0\right)-w^{\prime}\left(a_{k}-0\right)
$$


one can consider $\theta_{k}$ as a generalized coordinate and $M_{k}$ as the generalized force $M_{k}=M\left(a_{k}\right)$. It is known in the analytical mechanics that

$$
\theta_{k}=C_{o k} M_{k},
$$

for $k=1, \ldots, n$. In $(25) C_{o k}$ represents the additional compliance due to the crack (defect) located at the cross section $x=a_{k}, k=1, \ldots, n$. It is already shown by many researchers $[5,6,19,21]$ that

$$
C_{o k}=\frac{72 \pi\left(1-\nu^{2}\right)}{E b h_{o k}^{2}} f\left(s_{k}\right) .
$$

In (26) $h_{o k}=\min \left(h_{k-1}, h_{k}\right)$ and $s_{k}=\frac{c_{k}}{h_{o k}}$, whereas $\nu$ stands for the Poisson ratio. The function $f\left(s_{k}\right)$ in $(26)$ is defined as

$$
f\left(s_{k}\right)=\int_{0}^{s_{k}} y F^{2}(y) d y
$$

where $F=F\left(s_{k}\right)$ represents the stress correction function which can be specified by the interpolation of experimental data. Efforts have been made by many researchers to develop the best experimental approximation for calculation of the stress intensity factor. A review of the results is presented in the handbook by Tada et al. [31]. Following these results, one can use the correction function $F\left(s_{k}\right)$ as

$$
F\left(s_{k}\right)=\frac{\sqrt{\tan \pi \frac{s_{k}}{2}}}{\pi \frac{s_{k}}{2}} \cdot \frac{0.923+0.199\left(1-\sin \pi \frac{s_{k}}{2}\right)^{4}}{\cos \pi \frac{s_{k}}{2}} .
$$

Freund and Herrmann [17] suggested a shape correction function for larger cracks as

$F\left(s_{k}\right)= \begin{cases}1.99-2.47 s_{k}+12.97 s_{k}^{2}-23.17 s_{k}^{3}+24.8 s_{k}^{4}, & 0<s_{k}<0.5, \\ 0.663\left(1-s_{k}\right)^{-3 / 2}, & 0.5<s_{k}<1 .\end{cases}$

It is important to note that the correction functions for specimens of different shapes loaded in different manner should have different forms. Similarly, the type of crack (an intrinsic, a surface crack or an array crack) plays an important role in defining the shape of correction function [31].

Thus, it follows from (24)-(27) that the jump of the slope of the displacement can be defined as

$$
w^{\prime}\left(a_{k}+0\right)=w^{\prime}\left(a_{k}-0\right)-C_{o k}\left(E I_{k}-\eta P\right) w^{\prime \prime}\left(a_{k}+0\right),
$$

for $k=1, \ldots, n$, where $C_{o k}$ is defined by (25)-(29) and $I_{k}$ is defined by (7).

\section{Critical buckling load}

Consider the case of one-stepped nanobeam clamped at both ends. For the one-stepped nanobeam, according to (14)

$$
w=A_{o} \cos \lambda_{o} x+B_{o} \sin \lambda_{o} x+C_{o} x+D_{o}, x \in(0, a),
$$




$$
w=A_{1} \cos \lambda_{1} x+B_{1} \sin \lambda_{1} x+C_{1} x+D_{1}, x \in(a, l) .
$$

The boundary conditions (15) with the equality (31) furnish the relations

$$
D_{o}=-A_{o}, \quad C_{o}=-\lambda_{o} B_{o} .
$$

The support conditions (16) with (32) result in

$$
\begin{aligned}
A_{1} \cos \lambda_{1} l+B_{1} \sin \lambda_{1} l+C_{1} l+D_{1} & =0, \\
\lambda_{1}\left(-A_{1} \sin \lambda_{1} l+B_{1} \cos \lambda_{1} l\right)+C_{1} & =0,
\end{aligned}
$$

which leads to the relations

$$
\begin{aligned}
C_{1} & =\lambda_{1}\left(A_{1} \sin \lambda_{1} l-B_{1} \cos \lambda_{1} l\right), \\
D_{1} & =-A_{1}\left(\cos \lambda_{1} l+l \lambda_{1} \sin \lambda_{1} l\right)+B_{1}\left(l \lambda_{1} \cos \lambda_{1} l-\sin \lambda_{1} l\right) .
\end{aligned}
$$

The substitution of (33) and (34) in (31) and (32) presents the deflection of the nanobeam in the form

$$
w=A_{o}\left(\cos \lambda_{o} x-1\right)+B_{o}\left(\sin \lambda_{o} x-\lambda_{o} x\right)
$$

for $x \in(0, a)$ and

$$
\begin{aligned}
w= & A_{1}\left(\cos \lambda_{1} x+\lambda_{1} x \sin \lambda_{1} l-\cos \lambda_{1} l-l \lambda_{1} \sin \lambda_{1} l\right) \\
& +B_{1}\left(\sin \lambda_{1} x-\lambda_{1} x \cos \lambda_{1} l-\sin \lambda_{1} l+l \lambda_{1} \cos \lambda_{1} l\right)
\end{aligned}
$$

for $x \in(a, l)$.

The displacement $w=w(x)$ is continuous at $x=a$ if (21) is satisfied,

$$
\begin{aligned}
A_{1}\left(\cos \lambda_{1} a\right. & \left.+\lambda_{1} a \sin \lambda_{1} l-\cos \lambda_{1} l-l \lambda_{1} \sin \lambda_{1} l\right)+B_{1}\left(\sin \lambda_{1} a-\lambda_{1} a \cos \lambda_{1} l\right. \\
-\sin \lambda_{1} l & \left.+l \lambda_{1} \cos \lambda_{1} l\right)-A_{o}\left(\cos \lambda_{o} a-1\right)-B_{o}\left(\sin \lambda_{o} a-\lambda_{o} a\right)=0 .
\end{aligned}
$$

The continuity of the bending moment $M$ and the shear force $Q$ is verified by making use of (22) and (23) as

$$
\begin{aligned}
& \lambda_{1}^{2}\left(\eta P-E I_{1}\right)\left(A_{1} \cos \lambda_{1} a+B_{1} \sin \lambda_{1} a\right)-\lambda_{o}^{2}\left(\eta P-E I_{o}\right)\left(A_{o} \cos \lambda_{o} a\right. \\
& \left.+B_{o} \sin \lambda_{o} a\right)=0,
\end{aligned}
$$

and

$$
\begin{aligned}
& \lambda_{1}^{3}\left(\eta P-E I_{1}\right)\left(-A_{1} \sin \lambda_{1} a+B_{1} \cos \lambda_{1} a\right)-\lambda_{o}^{3}\left(\eta P-E I_{o}\right)\left(-A_{o} \sin \lambda_{o} a\right. \\
& \left.+B_{o} \cos \lambda_{o} a\right)=0 .
\end{aligned}
$$

The jump conditions (30) lead to the equation

$$
\begin{aligned}
& A_{1} \lambda_{1}\left(-\sin \lambda_{1} a+\sin \lambda_{1} l-\lambda_{1} C_{o 1}\left(E I_{1}-\eta P\right) \cos \lambda_{1} a\right)+B_{1} \lambda_{1}\left(\cos \lambda_{1} a\right. \\
& \left.-\cos \lambda_{1} l-\lambda_{1} C_{o 1}\left(E I_{1}-\eta P\right) \sin \lambda_{1} a\right)+A_{o} \lambda_{o} \sin \lambda_{o} a \\
& -B_{o} \lambda_{o}\left(\cos \lambda_{o} a-1\right)=0 .
\end{aligned}
$$

The system (35)-(38) is a linear algebraic system with respect to $A_{o}, B_{o}$ and $A_{1}, B_{1}$. Equalizing its determinant $\Delta$ to zero, one calculates the eigenvalues $\lambda_{o}$ and $\lambda_{1}$, which leads to calculating the values of critical buckling loads for clamped nanobeams by taking (13) into account. 
The critical buckling loads for simply supported and cantilever nanobeams can be determined by following the papers by Arif and Lellep [5, 6]. The boundary conditions for simply supported and cantilever nanobeams are presented by (17)-(20).

\section{Numerical results}

Calculations are carried out by considering the nanobeams/plates of constant thickness and one stepped nanobeams/plates of length $l$ having a crack of length $c$ at the step location $a$, uniformly penetrated throughout the thickness $h_{1}$ of the stepped column of the nanobeams. Nanobeams with various boundary conditions are taken into account for calculating the values of critical buckling loads $P_{c r}$ and critical stresses $\sigma_{c r}$ to solve the stability problems. Influence of discontinuities like steps, cracks and various boundary conditions on the stability of nanobeams is investigated as well as the impact of physical and geometrical parameter on the sensitivity of critical buckling load is studied here. Clamped (C-C), simply supported (S-S) and cantilever (C-F) nanobeams are investigated for the calculations. Results are shown in the Figures 3-9 and in Tables 1-4. Whereas, in Figures 10-12, the comparison of the results obtained in the current paper with those obtained by Wang et al. [35] is presented.

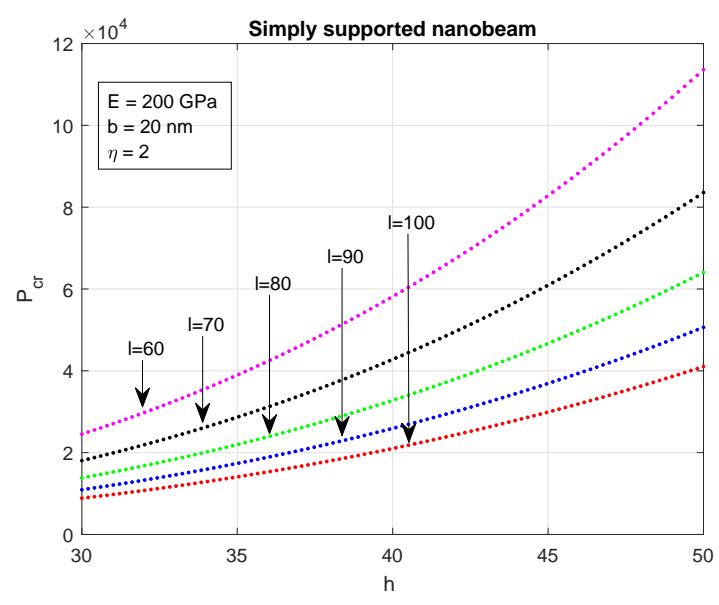

FiguRE 3. Critical buckling loads of simply supported nanobeams.

In Figures 3-5, the relationship between the critical buckling load $P_{c r}$ and the thickness $h$ of the nanobeam without a step and a crack is shown for nanobeams of different lengths. Various boundary conditions are applied to solve the problems of buckling. It can be seen from Figures $3-5$, that the values of the critical buckling load increase with increasing the thickness 
of the nanobeam and the values of the critical buckling load decrease by increasing the length of the nanobeams. Another observation to be mentioned here is the impact of boundary conditions on the values of the critical buckling loads of nanobeams. Calculations are carried out by fixing the same physical parameters for nanobeams with various boundary conditions. It can be seen that the values of the critical buckling loads are the highest in the case of clamped nanobeams whereas the cantilever nanobeams are much weaker than the clamped and simply supported nanobeams. The values of critical buckling loads for cantilever nanobeams are the lowest ones for these cases.

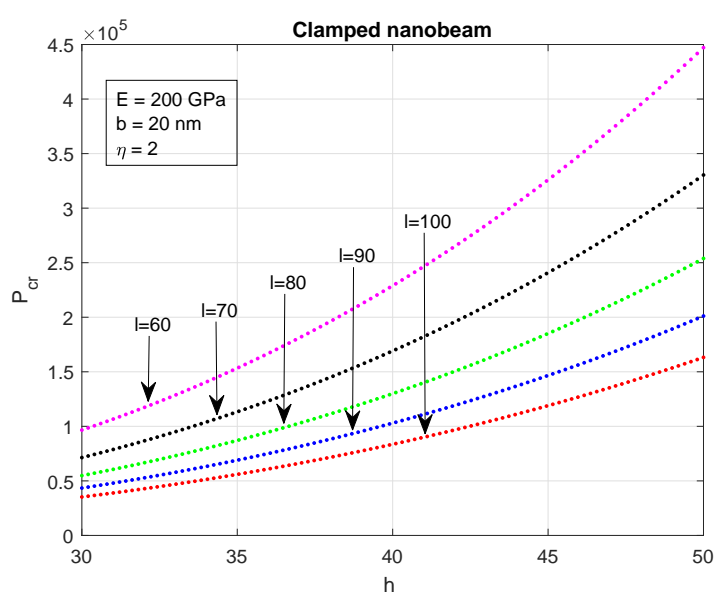

Figure 4. Critical buckling loads of clamped nanobeams.

Figures $6-8$ reveal the effect of boundary conditions and physical parameters like length $l$ and Young's modulus $E$ on the values of critical stresses $\sigma_{c r}$ of the nanobeams without a step and a crack. Results presented in Figures 6-8 show the strong impact of Young's modulus on the values of critical stresses for nanobeams with various boundary conditions. The values of the critical stresses increase significantly by increasing the value of Young's modulus and the values of the critical stresses decrease with increasing the length of the nanobeams. Additionally, it can be seen that the values of critical stresses are higher in the case of clamped nanobeams and these are the lowest in the case of cantilever nanobeams. 


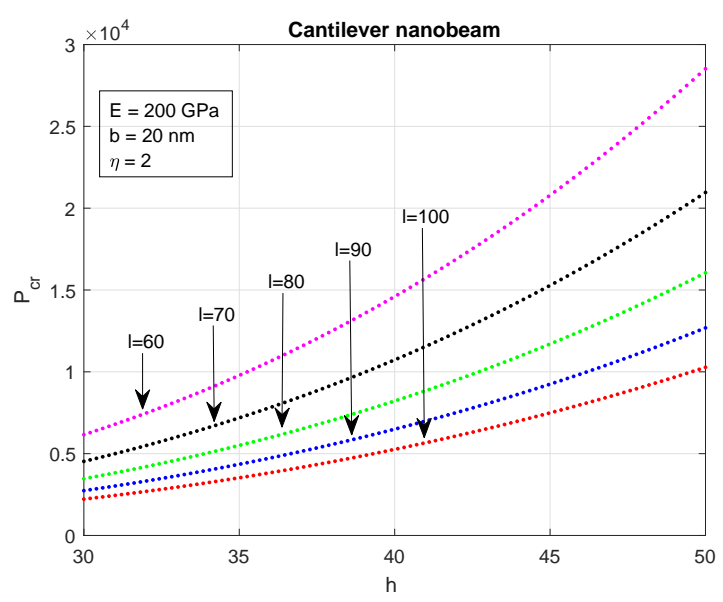

FiguRE 5. Critical buckling loads of cantilever nanobeams.

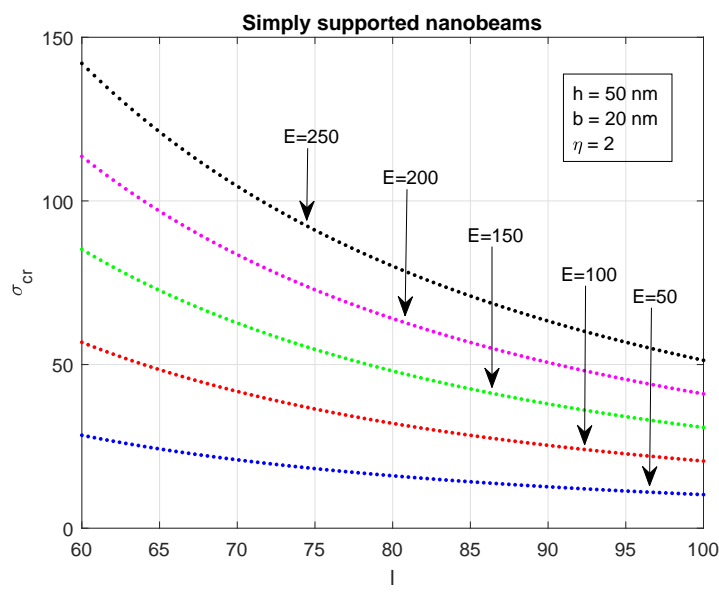

FiguRE 6. Critical stresses of simply supported nanobeams.

Critical buckling loads $P_{c r}$ of nanobeams of constant thickness as well as of stepped nanobeams including a crack at the step location are presented in Tables $1-4$. Nanobeams of length $l=100 \mathrm{~nm}$ and width $b=20 \mathrm{~nm}$ are considered in the numerical experiments, whereas the value of Young's modulus is fixed as $E=200 G P a$. Tables $1-4$ reveal the fact that the values of critical buckling loads for clamped nanobeams are always higher than those of simply supported and cantilever nanobeams. The values of critical buckling loads for cantilever nanobeams are always smaller than those of simply supported and clamped nanobeams. In Tables 1, 2 results are presented for nanobeams without a step and a crack. Table 1 reveals the impact of 


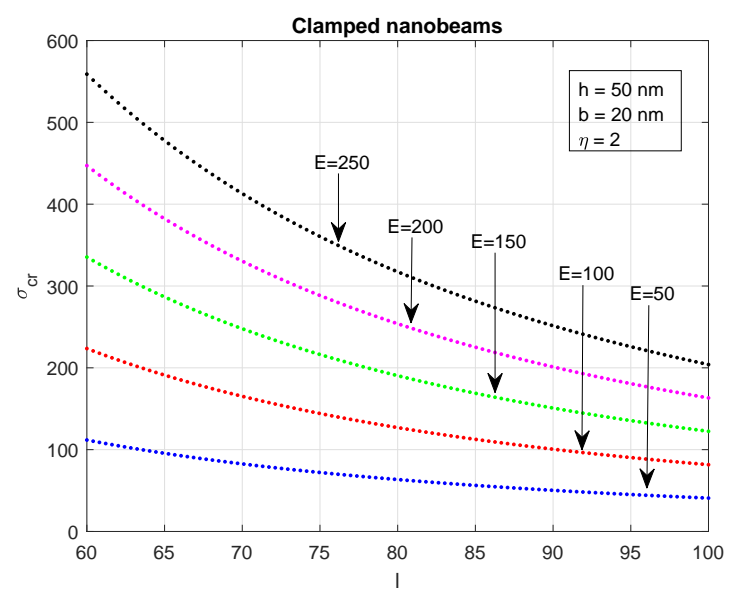

Figure 7. Critical stresses of clamped nanobeams.

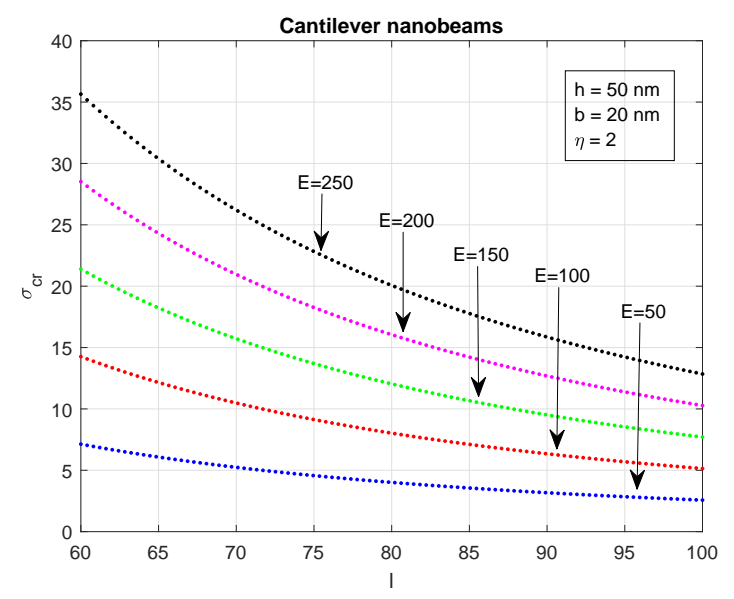

FiguRE 8. Critical stresses of cantilever nanobeams.

height to length ratio on the critical buckling loads of nanobeams with various boundary conditions. It can be seen that the value of critical buckling loads decreases monotonically by decreasing the value of height to length ratio of the nanobeam/plate. Table 2 presents the relationship between the non-dimensional length scale parameter $\eta$ and the critical buckling loads $P_{c r}$ of nanobeam/plate with various boundary conditions. One can see that the higher is the value of the nonlocal parameter, the smaller will be the value of the critical buckling load. Although the value of the nonlocal parameter has a little impact on the critical buckling loads of nanobeams, in the case 
TABLE 1. Impact of height to length ratio $h / l$ on the critical buckling loads of nanobeams with various boundary conditions.

\begin{tabular}{clll}
\hline$h / l$ & Clamped & Simply supported & Cantilever \\
\hline 0.5 & $1.6320 \mathrm{e}+05$ & $4.1042 \mathrm{e}+04$ & $1.0276 \mathrm{e}+04$ \\
0.4 & $8.3561 \mathrm{e}+04$ & $2.1014 \mathrm{e}+04$ & $5.2612 \mathrm{e}+03$ \\
0.3 & $3.5252 \mathrm{e}+04$ & $8.8651 \mathrm{e}+03$ & $2.2196 \mathrm{e}+03$ \\
0.2 & $1.0445 \mathrm{e}+04$ & $2.6267 \mathrm{e}+03$ & 657.6491 \\
\hline
\end{tabular}

TABLE 2. Relationship of non-dimensional length scale parameter $\eta$ to the critical buckling loads of nanobeams with various boundary conditions.

\begin{tabular}{llll}
\hline$\eta$ & Clamped & Simply supported & Cantilever \\
\hline 1 & $8.3889 \mathrm{e}+04$ & $2.1034 \mathrm{e}+04$ & $5.2625 \mathrm{e}+03$ \\
2 & $8.3561 \mathrm{e}+04$ & $2.1014 \mathrm{e}+04$ & $5.2612 \mathrm{e}+03$ \\
3 & $8.3235 \mathrm{e}+04$ & $2.0993 \mathrm{e}+04$ & $5.2599 \mathrm{e}+03$ \\
4 & $8.2911 \mathrm{e}+04$ & $2.0972 \mathrm{e}+04$ & $5.2586 \mathrm{e}+03$ \\
\hline
\end{tabular}

of nano-structures one can never neglect the impact of nonlocal length scale parameter while solving the static or dynamic stability problems.

TABLE 3. Effect of height to length ratio $h_{1} / l$ of the stepped column on the critical buckling loads of nanobeams with various boundary conditions.

\begin{tabular}{llll}
\hline$h_{1} / l$ & Clamped & Simply supported & Cantilever \\
\hline 0.4 & $2.6279 \mathrm{e}+4$ & $1.9148 \mathrm{e}+3$ & 50.7417 \\
0.3 & $2.9668 \mathrm{e}+3$ & $5.3561 \mathrm{e}+2$ & 28.5147 \\
0.2 & $1.7869 \mathrm{e}+3$ & 14.5270 & 12.6488 \\
0.1 & $2.3080 \mathrm{e}+2$ & 9.1770 & 3.1440 \\
\hline
\end{tabular}

In Tables 3, 4 results are presented for one stepped nanobeams including a crack at the step location. Here the height-to-length ratio of the first step is taken as $h_{o} / l=0.5$, the value of the Poisson ratio as $\nu=0.38$, and the crack length as $s=0.6$. Table 3 reveals the effect of the height-tolength ratio of the stepped column on the value of critical buckling loads of nanobeams with various boundary conditions. One can see that the value of the critical buckling loads monotonically decreases with decreasing the height-to-length ratio of the stepped column. In Table 4 the relationship 
TABLE 4. Relationship of critical buckling loads to the crack locations of nanobeams with various boundary conditions.

\begin{tabular}{clll}
\hline$a / l$ & Clamped & Simply supported & Cantilever \\
\hline 0.4 & $2.6279 \mathrm{e}+4$ & $1.9148 \mathrm{e}+3$ & 50.7417 \\
0.3 & $1.1010 \mathrm{e}+4$ & $9.13232+2$ & 43.4723 \\
0.2 & $8.3906 \mathrm{e}+3$ & $5.3891 \mathrm{e}+2$ & 38.0203 \\
0.1 & $4.8331 \mathrm{e}+3$ & $3.2631 \mathrm{e}+2$ & 33.7798 \\
\hline
\end{tabular}

between the critical buckling load and the crack location of the nanobeams with various boundary conditions is presented. The height-to-length ratio of the stepped column is chosen as $h_{1} / l=0.4$. It can be seen that the values of the critical buckling loads decrease monotonically by decreasing the value of the crack location of the nanobeam. It can be observed that the impact of boundary conditions on the stability of stepped nanobeams with cracks remains the same as in the case of nanobeams of constant thickness.

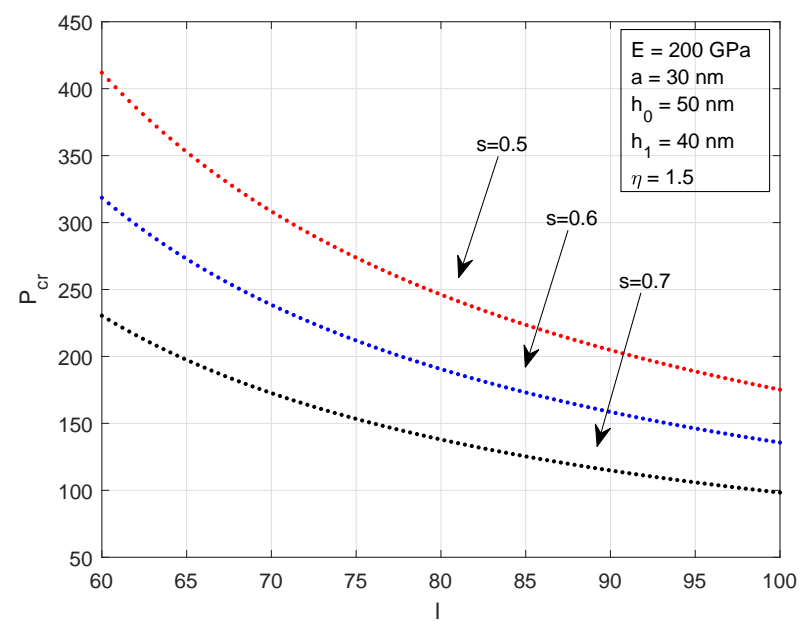

FiguRE 9. Impact of cracks on the critical buckling loads of cantilever nanobeams.

Figure 9 reveals the influence of crack length $s$ on the stability of a onestepped cantilever nanobeam. Figure 9 shows that the bigger is the value of crack length, the smaller is the critical buckling load of the stepped nanobeam. It also shows that the value of the critical buckling load for stepped nanobeams with cracks decreases with increasing the length of the nanobeam. 


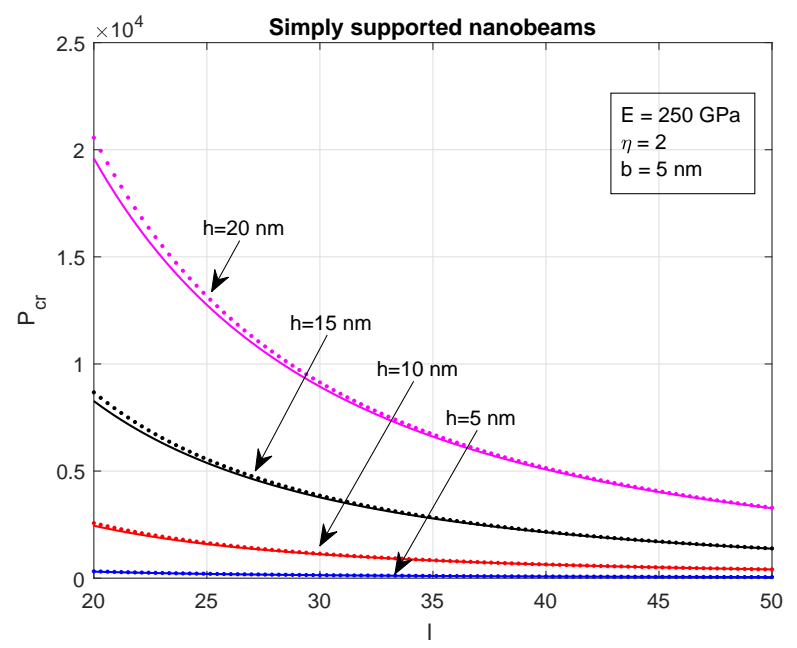

FiguRE 10. Comparison of results for simply supported nanobeams with Wang et al. [35].

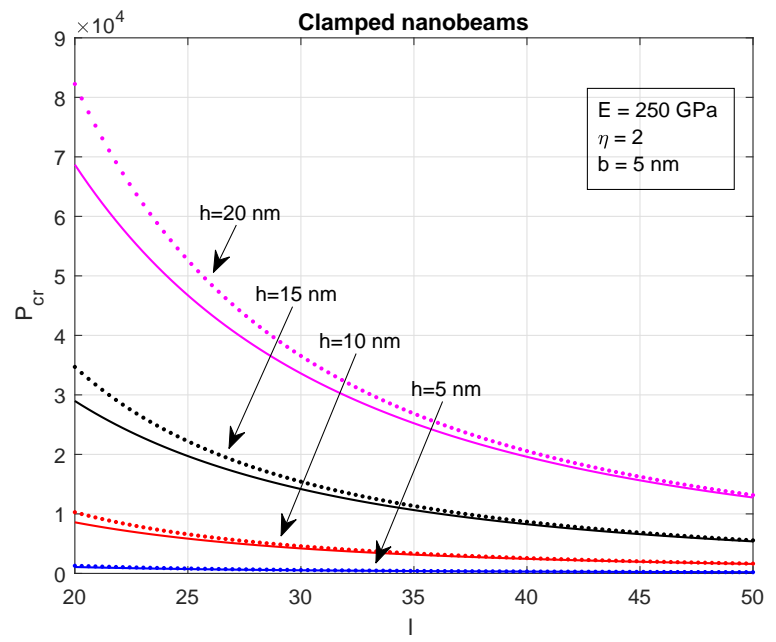

FiguRE 11. Comparison of results for clamped nanobeams with Wang et al. [35].

The results presented in the current article are compared with those obtained by Wang et al. [35] in Figures 10-12. Here the dotted lines correspond to the values of critical buckling loads of [35] and the solid lines correspond to the current solutions. It can be observed from Figures 10-12 that the results of the current paper are quite close to those obtained by Wang et al. [35]. 


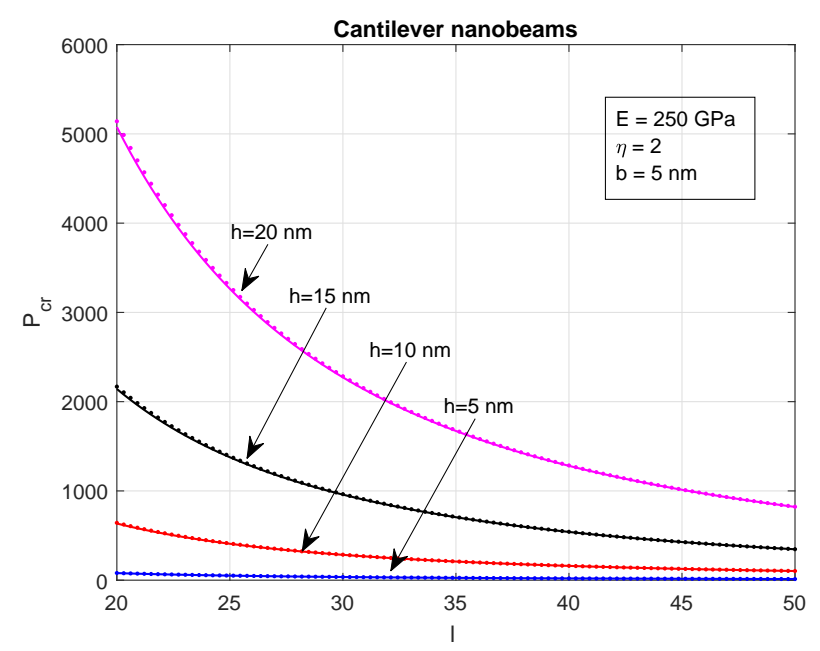

FiguRE 12. Comparison of results for cantilever nanobeams with Wang et al. [35].

\section{Conclusions}

The stability analysis of an axially loaded nanobeam/plate has been carried out. Stepped nanobeams with cracks at the re-entrant corners of the steps are considered here for the determination of critical buckling loads. An analytical method has been developed to investigate the effect of discontinuities like cracks, steps, and various boundary conditions on the stability of nanobeams. Calculations are carried out for simply supported, clamped and cantilever nanobeams. The effect of the nonlocal length scale parameter on the stability of nanobeams has been investigated. It is shown that the values of the critical buckling loads for clamped nanobeams are always higher than those of simply supported and cantilever nanobeams. Cantilever nanobeams provide the smallest values of the critical buckling loads in comparison to clamped and simply supported nanobeams. The effect of crack length and the crack location on the stability of stepped nanobeam has been investigated. It is also shown that the results of the present paper are close to those obtained by Wang et al. [35].

\section{Acknowledgements}

This research has been financed by the University of Tartu ASTRA Project PER ASPERA (European Regional Development Fund) and also the partial support from the Institutional Research Funding IUT 20-57 and Doctoral School of Mathematics and Statistics is gratefully acknowledged. 


\section{References}

[1] S. Adali, Variational formulation for buckling of multi-walled carbon nanotubes modelled as non-local Timoshenko beams, J. Theor. Appl. Mech. 50 (2012), 321-333.

[2] R. Ansari and S. Sahmoni, Bending behaviour and buckling of nanobeams including surface stress effect corresponding to different beam theories, Int. J. Eng. Sci. 49 (2011), $1244-1255$.

[3] B. Akgöz and Ö. Civalek, Analysis of micro-sized beams for various boundary conditions based on the strain gradient elasticity theory, Arch. Appl. Mech. 82 (2012), 423-443.

[4] B. Arash and Q. Wang, A review on the application of nonlocal elastic models in modelling of carbon nanotubes and graphenes, Comp. Mater. Science 51 (2012), 303313.

[5] H. Arif and J. Lellep, Buckling of nanobeams and nanorods with cracks, $13^{\text {th }}$ International Conference MBMST, Vilnius Lithuania, 16 - 17 May: VGTU Press (2019), $722-727$

[6] H. Arif and J. Lellep, Buckling analysis of cantilever nanobeams with defects, Appl. Nanosci. (2021), 1-7. https://doi.org/10.1007/s13204-021-01827-2.

[7] M. Aydogdu, A general nonlocal beam theory: its application to nanobeam bending, buckling and vibration, Physica E 41 (2009), 1651-1655.

[8] L. Behera and S. Chakraverty, Application of Differential Quadrature method in free vibration analysis of nanobeams based on various nonlocal theories, Computers and Mathematics with Applications 69(12) (2015), 1444-1462.

[9] S. M. Bagdatli, Non-linear vibration of nanobeams with various boundary conditions based on nonlocal elasticity theory, Composites Part B 80 (2015),43-52.

[10] N. Challamel, Variational formulation of gradient or/ and nonlocal higher-order shear elasticity beams, Composite Structures 105 (2013), 351-368.

[11] N. Challamel and I. Elishakoff, Surface stress effects may induce softening: EulerBernoulli and Timoshenko buckling solutions, Physica E 44 (2012), 1862-1867.

[12] A. D. Dimarogonas, Vibration of cracked structures: A state of the art review, Eng. Fract. Mech. 55 (1996), 831-857.

[13] S. Emam, A general nonlocal nonlinear model for buckling of nanobeams, Appl. Mathem. Modell. 37 (2013), 6929-6939.

[14] A. C. Eringen, Nonlocal Continuum Field Theories. Springer, New York, 2002.

[15] A. C. Eringen, On differential equations of nonlocal elasticity and solutions of screw dislocaton and surface waves, J. Appl. Phys. 54 (1983), 4703-4710.

[16] M. Farshad, Stability of Structures, Elsevier, 1994.

[17] L. B. Freund and G. Herrmann, Dynamic fracture of a beam or plate in plane bending, Trans. ASME 76 (1976), 112-116.

[18] D. Kumar, C. Heinrich, and A. M. Waas, Buckling analysis of carbon nanotubes modeled using nonlocal continuum theories, J. App. Phys. 103(7) (2008), 073521.

[19] J. Lellep and T. Kraav, Buckling of beams and columns with defects, Int. J. Struct. Stability and Dynamics 16 (2016), 10.1142.

[20] J.Lellep and A. Lenbaum, Free vibrations of stepped nano-beams, Int. J. Comp. Meth. Exp. Meas. 6 (2018), 716-725.

[21] J. Lellep and A. Liyvapuu, Natural vibrations of stepped arches with cracks, Argon. Res. 14 (2016), 821-830.

[22] J. Lellep and E. Sakkov, Buckling of stepped composite columns, Mech. Compos. Mater. 42 (2006), 63-72.

[23] C. Li, C. W. Lim, J. L. Yu, and Q. C. Zeng, Analytical solutions for vibration of simply supported nonlocal nanobeams with an axial force, Int. J. Stability and Dynamics 11 (2011), 257-271. 
[24] P. Lu, H. P. Lee, C. Lu, and P. Q. Zhang, Dynamic properties of flexural beams using a nonlocal elasticity model, J. Appl. Pys. 99 (2006), 073510.

[25] T. Murma and S. C. Pradhan, Small-scale effect on the vibration of nonuniform nanocantilever based on nonlocal elasticity theory, Physica E 41 (2009), 1628-1633.

[26] C. Polizzotto, Nonlocal elasticity and related variational principles, Int. J. Solids Structures 38 (2001), 7359-7380.

[27] H. Roostai and M. Haghpanahi, Vibration of nanobeams of different boundary conditions with multiple cracks based on nonlocal elasticity theory, Appl. Math. Modell. 38 (2014), 1159-1169.

[28] M. Skrinar, On critical buckling load estimation for slender transversely cracked beamcolumns by the application of a simple computational model, Comp. Mater. Sci. 43 (2008), 190-198.

[29] J. N. Reddy, Nonlocal theories for bending, buckling and vibration of beams, Int. J. Eng. Sci. 45 (2007), 288-307.

[30] J. N. Reddy, Nonlocal nonlinear formulations for bending of classical and shear deformation theories of beams and plates, Int. J. Eng. Sci. 48(11) (2010), 1507-1518.

[31] M. Tada, P. C. Paris, and G. R. Irwin, Stress Analysis of Cracks Handbook, ASME, New York, 2000.

[32] H. T. Thai, A nonlocal beam theory for bending, buckling and vibration of nanobeams, Int. J. Eng. Sci. 52 (2012), 56-64.

[33] C. M. Wang, C. Y. Wang, and J. N. Reddy, Exact Solutions for Buckling of Structural Members, CRC Press, Boca Raton, 2005.

[34] C. M. Wang, H. Zhang, N. Challamel, and W. H. Duan, On boundary conditions for buckling and vibration of nonlocal beams, European J. Mech. A/Solids 61 (2017), $73-81$.

[35] C. M Wang, Y. Y. Zhang, S. S. Ramesh, and S. Kitipornchai, Buckling analysis of micro and nano-rods/tubes based on nonlocal Timoshenko beam theory, J. Phys. D. Appl. Phys. 39 (2006), 3904-3909.

[36] Y. Y. Zhang, C. M. Wang, and N. Challamel, Bending, buckling, and vibration of micro/nanobeams by hybrid nonlocal beam model, J. Eng. Mech. 136(5) (2010), 562574 .

Institute of Mathematics and Statistics, University of Tartu, 51009 Tartu, ESTONIA.

E-mail address: hina.arif@ut.ee

E-mail address: jaan.lellep@ut.ee 